\title{
LEVAMISOLE IMPROVES HISTOMORPHOMETRIC PARAMETERS OF SMALL INTESTINAL WALL OF BROILER CHICKENS
}

\author{
T. SHOMALI ${ }^{1}$, S. HAMEDI ${ }^{2} \&$ H. SOLHDOOST ${ }^{2}$ \\ ${ }^{1}$ Division of Pharmacology and Toxicology, Department of Basic Sciences, \\ School of Veterinary Medicine, Shiraz University, Shiraz, Iran; ${ }^{2}$ Department \\ of Basic Sciences, College of Veterinary Medicine, Islamic Azad University, \\ Karaj Branch, Alborz, Iran
}

\section{Summary}

Shomali, T., S. Hamedi \& H. Solhdoost, 2017. Levamisole improves histomorphometric parameters of small intestinal wall of broiler chickens. Bulg. J. Vet. Med., 20, No 4, 385-391.

\begin{abstract}
Sixty one-day old chickens were divided into 6 equal groups and treated with $0,2,5,10,15$ and 25 $\mathrm{mg} / \mathrm{kg}$ levamisole from day 1 to 45 . Then, all birds sacrificed and samples were taken from duodenum, jejunum and ileum. Cross-sections were made and H\&E stained. Histomorphometric parameters including villus height, crypt depth, villus width, sub mucosal width, muscular layer width and the villus height/crypt depth ratio were determined. Duodenal villi became wider in all levamisole treated groups but only the highest dose resulted in taller villi. Jejunal villi became taller without significant change in their width. This was accompanied by a decrease in crypt depth and increased villus height/crypt depth ratio in all treated groups. In ileum, only birds treated with the highest dose had higher villus height, although levamisole at all doses resulted in wider villi. Sub mucosal width increased in birds treated with 15 and $25 \mathrm{mg} / \mathrm{kg}$ levamisole. In conclusion, levamisole can improve histomorphometric parameters of small intestinal wall of broiler chickens. This can partly explain the mechanism for previously described positive effects of levamisole on performance of broilers.
\end{abstract}

Key words: broilers, histology, levamisole hydrochloride, small intestine

Improving performance parameters of birds and reducing the costs of disease prevention and/or treatment results in economically significant outcomes in broiler industry. Traditionally, synthetic chemicals and antibiotics have been used to prevent or treat poultry diseases and enhance performance with at least partial success. Achieving an optimal growth performance includes balanced nutrition, proper management and prophylactic measures against diseases, for example, by the use of immunostimulants.

Levamisole (LMS) is the levo isomer of ditetramisole, which is a racemic mixture. It is widely used in cattle, sheep, goats, swine and poultry and is effective against lung worms and gastrointestinal 
nematodes. This agent is currently being used to treat capillaria infection in poultry, and its recommended anthelmintic dosage in poultry is $25 \mathrm{mg} / \mathrm{kg}$ body weight. Different researchers have shown positive effects of LMS on immune responses of avian species (Habibi et al., 2012; Oladele et al., 2012; Shomali et al., 2012). On the other hand, in a study performed by Porchezhian \& Punniamurthy (2006) LMS given orally in different dose levels to broilers had positive effect on performance parameters including reduced cumulative feed intake and enhanced cumulative feed efficiency as well as cumulative body weight gain.

Morphological changes of intestines are important in determining animal performance due to changes in the absorptive area and absorptive capacity; they affect digestion and absorption of nutrients. In fact, intestinal morphology is a main indicator of gut health and its functional status. Small intestine, especially crypts and villi of the absorptive epithelium, play a significant role in the final phase of nutrient digestion and absorption (Wang \& Peng, 2008). Intestinal development can be evaluated through measurements of the crypt, a region in which new intestinal cells are formed, as well as villus height and surface area, to determine the area available for digestion and absorption (Swatson et al., 2002; Franco et al., 2006).

As far as we know, there is no previous study on the effect of LMS administration on intestinal histomorphometric parameters of broilers. This along with previous reports about the positive effects of LMS on broilers' performance; motivated us to evaluate the possible effects of this agent on histomorphometric parameters of intestinal wall of normal broilers which can be helpful in elucidation of its mechanism for improving feed efficiency in broilers.

Sixty one-day old chickens (Arbor Acres) were housed individually in cages in a temperature-controlled room (24 \pm $2{ }^{\circ} \mathrm{C}$ ) with $16: 8 \mathrm{~h}$ light/dark cycle. Each cage was fitted with an individual feeder and a nipple drinker. Birds had free access to food and tap water during the experimental period. Routine vaccination programs against ND, IB and AI were followed during the rearing period including live vaccine against $\mathrm{ND}+\mathrm{H} 120$ on day one via eye drop, B1 via eye drop and oilemulsified ND+AI vaccine by SC route on day 7, H120 via eye drop on day 14 and La Sota via eye drop on day 21. Birds were randomly divided into six equal groups ( $\mathrm{n}=10$ each) on day one. Daily water intake of birds was calculated and birds in different groups were treated with 0 (control group), 2, 5, 10, 15 and 25 $\mathrm{mg} / \mathrm{kg}$ body weight levamisole (Damloran Razak Pharma Co., Iran) in drinking water from day 1 to 45 .

At the end of the experiment (day 46), all birds were sacrificed by cervical dislocation. Whole small intestine was removed immediately and immersed in $10 \%$ buffered formalin. After fixation, $1 \mathrm{~cm}$ thick samples were taken from the middle parts of duodenum, jejunum and ileum. The intestinal segmentation was according to the Samanya \& Yamauchi (2002): duodenum (from the gizzard to pancreatic and bile duct), jejunum (from the bile duct to Meckel's diverticulum) and ileum (from the Meckel's diverticulum to ileo-caecalcolonic junction). Routine histological laboratory methods including dehydration, clearing and paraffin embedding used and paraffin blocks made. Six $\mu$ m-thick transverse cross-sections were made by a rotary microtome, a total number of $10 \mathrm{sec}-$ tions used from each intestinal segment of 
each bird; sections stained with H\&E for measuring histomorphometric parameters including villus height from the tip of the villus to the crypt, crypt depth from the base of the villi to the sub mucosa, villus width, sub mucosal width, muscularis from the submucosa to the external layer of the intestine, and the villus height/crypt depth ratio (Geyra et al., 2001) as shown in Fig. 1A, by using a linear graticule under light microscope. All parameters were measured as the arithmetic mean of 15 measurements of each parameter per section. All methods used in the present study are in accordance with internationally accepted guidelines for care and use of animals in experiments.

Data were expressed as mean \pm SD. Data comparisons performed by one-way ANOVA followed by Tukey's multiple comparison test and differences considered statistically significant at $\mathrm{P}<0.05$.

In general, health condition of birds remained satisfactory throughout the experiment.

As shown in photomicrographs, duodenal villi became significantly wider in all LMS-treated groups as compared to control (Fig. 1A, 1B); however, except for the highest dose which resulted in taller villi, other doses had no appreciable effect on height of villi. The highest dose also had the most profound effect on increasing the muscular layer width.

In contrary to duodenum, jejunal villi became taller without significant change in their width due to LMS administration. The increase in villi length was accompanied by a decrease in crypt depth. These changes were clearly reflected in an appreciably increased villus height/crypt depth ratio in all treated groups. Sub mucosal and muscular widths were not affected by LMS administration (Fig. 2).
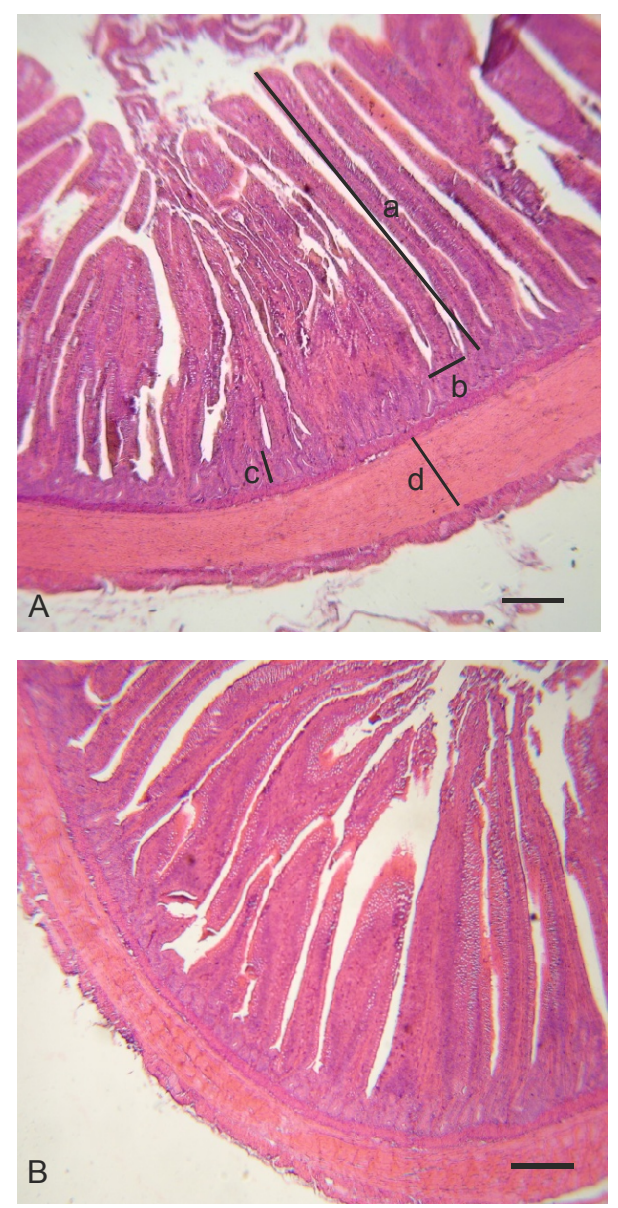

Fig. 1. A. Duodenal wall of a control bird: villus height (a), villus width (b), crypt depth (c) and muscular layer width (d). B. Duodenal wall of a chicken treated at $25 \mathrm{mg} / \mathrm{kg}$ LMS: villi are wider and taller as compared to control. H \& E; bar $=1 \mu \mathrm{m}$.

In ileum, except for birds that were treated with the highest dose, other treated groups had statistically similar villus height compared to control group. The change in villi width was more noteworthy and LMS at all doses resulted in wider villi. Sub mucosal width significantly increased in birds treated with 15 and 25 $\mathrm{mg} / \mathrm{kg}$ LMS in comparison with control 

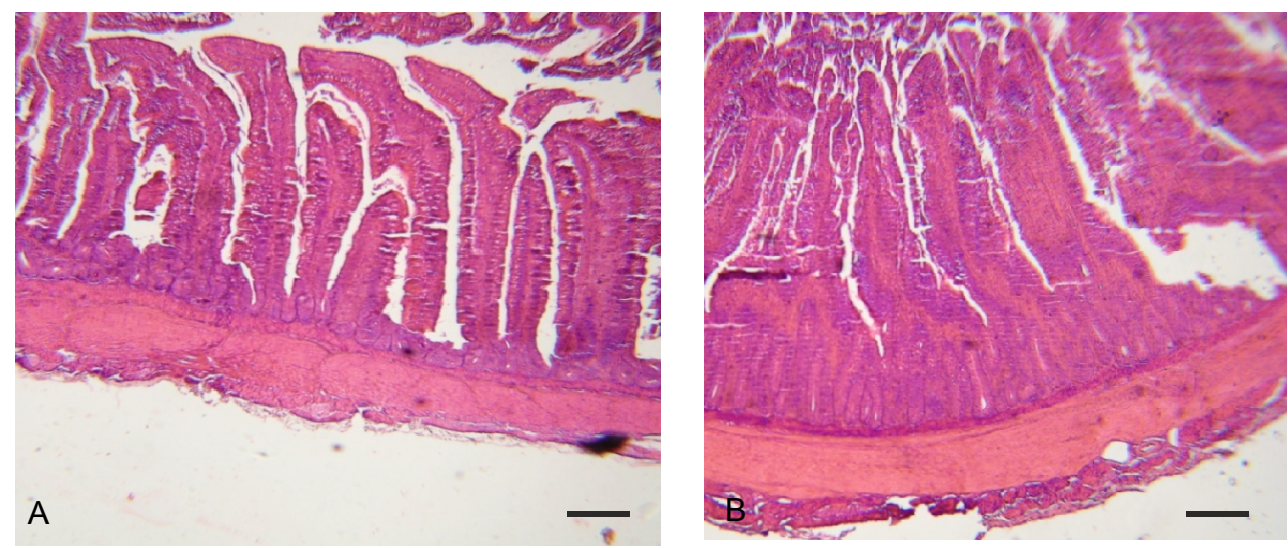

Fig. 2. Jejunal wall of a control bird (A) and of a chicken treated at $25 \mathrm{mg} / \mathrm{kg}$ LMS (B). Villi became taller without significant change in their width due to LMS administration. The increase in villi length was accompanied by a decrease in crypt depth. $\mathrm{H} \& \mathrm{E} ; \mathrm{bar}=1 \mu \mathrm{m}$.
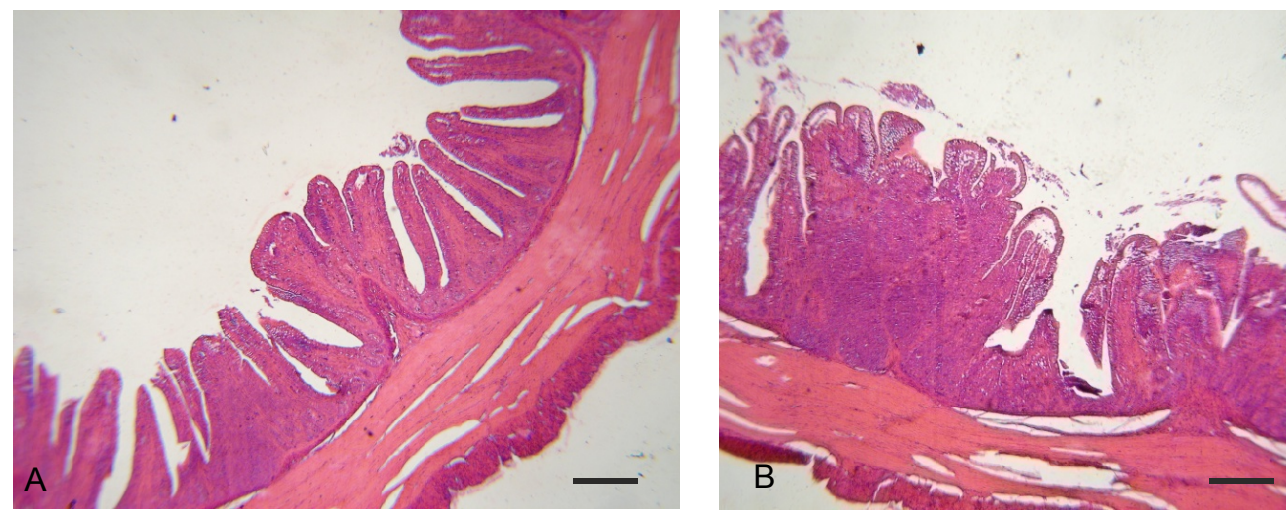

Fig. 3. Ileal wall of a control bird (A) and of a chicken treated at $25 \mathrm{mg} / \mathrm{kg}$ LMS (B). The increase in ileal submucosal width after LMS treatment due to expansion of disseminated and nodular lymphatic tissue is noteworthy. $\mathrm{H} \& \mathrm{E}$; bar $=1 \mu \mathrm{m}$.

birds; this increase was due to expansion of lymphatic tissues. Other parameters remained statistically the same among different groups (Fig. 3). All histomorphometric data are summarised in Table 1.

LMS is a synthetic compound that has been classically used as anthelmintic agent in animals. Moreover, LMS has proven immune stimulants properties (Cuesta et al., 2002). As mentioned above, some researchers have reported LMS as an im- munomodulator, increasing performance parameters of birds. This motivated us to evaluate the possible changes in histomorphometric parameters intestinal wall of broilers that received LMS, as a plausible mechanism for reported desirable effects of the LMS on performance of these birds. In fact, lengthening of villi may increase total luminal villus absorptive area and subsequently result in satisfactory digestive enzyme action and higher 


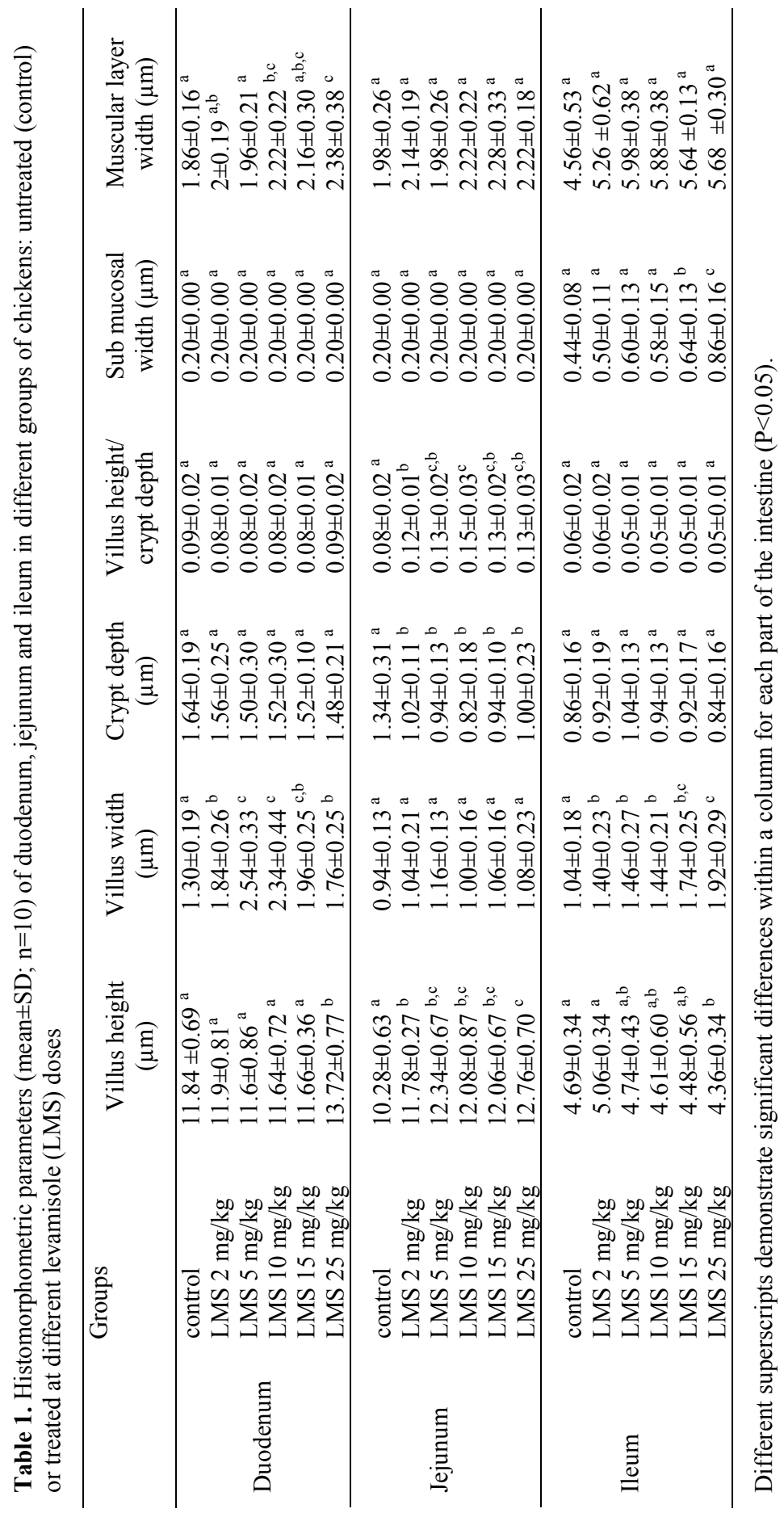


transport of nutrients at the villus surface (Tufarelli et al., 2010). Crypts are considered as the villus factory and deeper crypts indicate fast tissue turnover to permit renewal of the villus as needed in response to normal sloughing or inflammation from pathogens or their toxins and high demands for tissue (Yason et al., 1987). A shortening of the villi and deeper crypts may lead to poor nutrient absorption, increased secretion in the gastrointestinal tract, and lower performance $(\mathrm{Xu}$ et al., 2003). In contrast, increase in villus height and villus height/crypt depth ratio is directly correlated with increase in epithelial cell turnover (Fan et al., 1997) and longer villi are associated with activated cell mitosis (Samanya \& Yamauchi, 2002). In another word, villus height/crypt depth ratio is a valuable criterion for estimating the digestive capacity of the small intestine and a decrease in this ratio is considered to be deleterious to digestion and absorption, and vice versa (Pluske et al., 1997). In the present study, a significant increase in villus height/crypt depth ratio was observed in jejunal mucosa of treated birds. Since jejunum is where maximum digestion and absorption takes place because of large luminal site and presence of more mature enterocytes (Samanya et al., 2002), this finding may be important in describing the positive effects of LMS on performance in previous study (Porchezhian \& Punniamurthy, 2006). Moreover in duodenum and ileum of treated birds villi became broader by keeping the same height as compared to control. It is clear that wider villi have more surface area for absorption.

In our study, we observed an increase in muscularis width in duodenum of some groups treated with LMS. Thicker muscularis can possibly be accompanied with stronger movements which facilitate transportation of substances to jejunum. As previously stated, there was a significant increase in ileal submucosal width of LMS treated birds. This increase was mainly due to expansion of more developed lymphatic tissue including both disseminated and nodular lymphatic tissue in this part of the intestine which can be described by immunostimulant property of LMS.

The results of present study showed that administration of LMS (especially at its anthelmintic dose) can improve histomorphometric parameters of small intestinal wall of broiler chickens. This can partly explain the mechanism for previously described positive effects of LMS on body weight and performance of broilers.

\section{REFERENCES}

Cuesta, A., M. Esteban \& J. Meseguer, 2002. Levamisole is a potent enhancer of gilthead seabream natural cytotoxic activity. Veterinary Immunology and Immunopathology, 89, 169-174.

Geyra, A., Z. Uni \& D. Sklan, 2001. Enterocyte dynamics and mucosal development in the posthatch chick. Poultry Science, 80, 776-782.

Habibi, M., M. A., Zandieh, H. Ghahri, R. Salman Zadeh \& F. Yeganeh, 2012. Effects of levamisole on the immune response of broilers against Newcastle disease vaccines. African Journal of Pharmacy and Pharmacology, 6, 1860-1864.

Fan, Y., J. Croom, V. Christensen, B. Black, A. Bird, L. Daniel, B. McBride \& E. Eisen, 1997. Jejunal glucose uptake and oxygen consumption in turkey poults selected for rapid growth. Poultry Science, 76, 1738-1745.

Franco, J. R.G., A. E. Murakami, M. R. M. Natali, E. R. M. Garcia \& A. C. Furlan, 2006. Influence of delayed placement and 
dietary lysine levels on small intestine orphometrics and performance of broilers. Brazilian Journal of Poultry Science, 8, 233-241.

Oladele, O.A., B.O. Emikpe, C.A.O. Adeyefa \& F. Enibe, 2012. Effects of levamisole hydrochloride on cellular immune response and flock performance of commercial broilers. Brazilian Journal of Poultry Science, 14, 233-304.

Pluske, J. R., D. J. Hampson \& I. H. Williams, 1997. Factors influencing the structure and function of the small intestine in the weaned pig - a review. Livestock Production Science, 51, 215-236.

Porchezhian, T. \& N. Punniamurthy, 2006. Effect of oral levamisole hydrochloride on feed intake and body weight of broiler chicks. Journal of Animal and Veterinary Advances, 5, 847-848.

Samanya, M. \& K. Yamauchi, 2002. Histological alterations of intestinal villi in chickens fed dried Bacillus subtilis var. natto. Comparitive Biochemistry and Physiology Part A, 133, 95-104.

Shomali, T., N. Mosleh \& A. Alaeddini, 2012. Levamisole enhances cell-mediated immune responses and reduces shedding of H9N2 avian influenza virus in Japanese quails (Coturnix coturnix japonica). American Journal of Animal and Veterinary Sciences, 7, 16-20.

Swatson, H. K., R. Gous, P.A. Iji \& R. Zarrinkalam, 2002. Effect of dietary protein level, amino acid balance, and feeding level on growth, gastrointestinal tract, and mucosal structure of the small intestine in broiler chickens. Animal Research, 51, 501-515.
Tufarelli, V., S. Desantis, S. Zizza \& V. Laudadio, 2010. Performance, gut morphology, and carcass characteristics of fattening rabbits as affected by particle size of pelleted diets. Archive of Animal Nutrition, 64, 373-382.

Wang J. X. \& K. M. Peng, 2008. Developmental morphology of the small intestine of African ostrich chicks. Poultry Science, 87, 2629-2635.

Xu, Z. R., C. H Hu, M. S. Xia, X. A. Zhan \& M. Q. Wang, 2003. Effects of dietary fructooligosaccharide on digestive enzyme activities, intestinal microflora and morphology of male broilers. Poultry Science, 82, 1030-1036.

Yason, C. V., B. A. Summers \& K. A. Schat, 1987. Pathogenesis of rotavirus infection in various age groups of chickens and turkeys: Pathology. American Journal of Veterinary Research, 48, 927-938.

Paper received 20.04.2015; accepted for publication 15.06.2015

\section{Correspondence:}

Somayeh Hamedi

Department of Basic Sciences,

College of Veterinary Medicine,

Islamic Azad University,

Karaj Branch, Alborz, Iran

P.O. Box: 31485-313,

Tel: +982634182550,

Fax: +982634418156,

e-mail: sahar_hamedi@yahoo.com 